\title{
Enhancement of laser-driven ion acceleration in non-periodic nanostructured targets
}

\author{
J. Ferri ${ }^{\circledR 1} \uparrow$, I. Thiele ${ }^{1, \dagger}$, E. Siminos ${ }^{2}$, L. Gremillet ${ }^{\circledR 3}$, E. Smetanina ${ }^{2}$, \\ A. Dmitriev ${ }^{2}$, G. Cantono ${ }^{4}$, C.-G. Wahlström ${ }^{4}$ and T. Fülöp \\ ${ }^{1}$ Department of Physics, Chalmers University of Technology, SE-41296 Göteborg, Sweden \\ ${ }^{2}$ Department of Physics, Gothenburg University, SE-41296 Göteborg, Sweden \\ ${ }^{3}$ CEA, DAM, DIF, F-91297 Arpajon, France \\ ${ }^{4}$ Department of Physics, Lund University, SE-22100 Lund, Sweden
}

(Received 28 June 2019; revised 29 November 2019; accepted 2 December 2019)

Using particle-in-cell simulations, we demonstrate an improvement of the targetnormal-sheath acceleration (TNSA) of protons in non-periodically nanostructured targets with micron-scale thickness. Compared to standard flat foils, an increase in the proton cutoff energy by up to a factor of two is observed in foils coated with nanocones or perforated with nanoholes. The latter nano-perforated foils yield the highest enhancement, which we show to be robust over a broad range of foil thicknesses and hole diameters. The improvement of TNSA performance results from more efficient hot-electron generation, caused by a more complex laser-electron interaction geometry and increased effective interaction area and duration. We show that TNSA is optimized for a nanohole distribution of relatively low areal density and that is not required to be periodic, thus relaxing the manufacturing constraints.

Key words: intense particle beams, plasma simulation

\section{Introduction}

Laser-driven ion acceleration has become a well-established technique to produce compact, high-energy ion beams, owing to the ultra-strong accelerating fields that can be achieved at the surfaces of solid targets (Daido, Nishiuchi \& Pirozhkov 2012; Macchi, Borghesi \& Passoni 2013). Such ion sources show great potential for a number of applications ranging from radiography (Romagnani et al. 2005) to nuclear photonics (Habs et al. 2011) and proton therapy (Bulanov \& Khoroshkov 2002). However, even though proton energies close to $100 \mathrm{MeV}$ have been demonstrated in recent experiments using petawatt-class laser facilities (Wagner et al. 2016; Higginson et al. 2018), the few tens of $\mathrm{MeV}$ energies that are routinely attained using multi-terawatt-class laser systems are insufficient for many of the foreseen applications, therefore limiting the applicability of laser-driven ion sources. This spurs the development of novel schemes yielding significantly increased proton energies.

The most robust, and extensively investigated, acceleration scheme is the so-called target-normal-sheath acceleration (TNSA) (Snavely et al. 2000; Wilks et al. 2001),

$\dagger$ Email addresses for correspondence: julien.ferri@chalmers.se, illia-thiele@web.de 
whereby surface ions are driven outwards by the charge-separation field set up by the laser-accelerated relativistic electrons escaping into vacuum. Because of their large charge-to-mass ratio, the protons that are naturally present due to hydrogen-containing contaminants at the target surfaces respond the fastest to the electric sheath field, and reach the highest velocities. Their final energy spectrum has typically the form of a decreasing exponential with a sharp high-energy cutoff.

Different strategies have been explored in recent years to increase the proton cutoff energies resulting from TNSA. With micrometric foil targets, this requires enhancing the fast electron generation at the target front side. One option is to manipulate the laser temporal profile so as to create a preplasma with an optimal scale length (Kaluza et al. 2004; Nuter et al. 2008; Brenner et al. 2014), or to induce an optimal electromagnetic interference pattern (Ferri, Siminos \& Fülöp 2019). Another option is to modify the target properties: reduction of the target thickness (Neely et al. 2006; Ogura et al. 2012) or transverse size (Buffechoux et al. 2010) thus results in higher proton energies and numbers. An alternative, which is addressed in the present paper, is to employ nano- (or micro-) structured targets. Enhancement of the proton energy has been discussed for periodic nanohole targets (Nodera et al. 2008; Psikal et al. 2016), periodic nanobrush targets (Yu et al. 2012), targets with a symmetrical nanocone distribution (Brantov \& Bychenkov 2013) and grating surfaces (Andreev et al. 2011; Sgattoni et al. 2015; Andreev et al. 2016; Blanco et al. 2017). Besides, foils with periodic surface structures have been experimentally shown to yield a twofold increase in proton energy (Margarone et al. 2012; Ceccotti et al. 2013). However, little attention has been paid so far to the potential of non-periodic structures, even if the addition of nanospheres with irregular diameters on the front side of the target has been studied (Klimo et al. 2011), as have recently been irregularities in grating targets (Blanco, Flores-Arias \& Vranic 2019) and surfaces coated with foams or nanowires (Zigler et al. 2013; Fedeli et al. 2018). Importantly, relaxing the constraint on the structure periodicity would enable simpler and more robust target fabrication methods (Langhammer, Kasemo \& Zoric 2007; Zigler et al. 2011), as is required to bring laser-driven proton sources closer to applications.

In this paper, we investigate by means of particle-in-cell (PIC) simulations the potential of non-periodically structured targets to enhance TNSA. Two target types are considered, consisting of a flat foil either coated on the front surface with randomly positioned nanocones ('nanocone targets') or perforated by nanoholes ('nanohole targets'). In both cases, the proton cutoff energy is increased by up to a factor of two compared with flat foils. The causes of this improvement are a more complex interaction geometry, combined with locally enhanced charge-separation fields, and a larger effective laser-matter interaction area.

Nanohole targets which, in the case of relatively low areal density, yield the highest enhancement, further lead to efficient electrostatic confinement, hence lengthening the interaction duration and sustaining a high hot-electron density. In $\S 2$, we describe the physical and numerical set-up and in $\S 3$ we compare the results obtained with the structured targets to the flat-foil case and investigate the origin of proton energy enhancement. In $\S 4$, a parametric study of the nanohole targets is presented. Finally, we summarize our results in $\S 5$.

\section{Physical and numerical set-ups}

We will investigate how the aforementioned two types of structured foils behave with respect to proton acceleration by means of two-dimensional PIC simulations with 

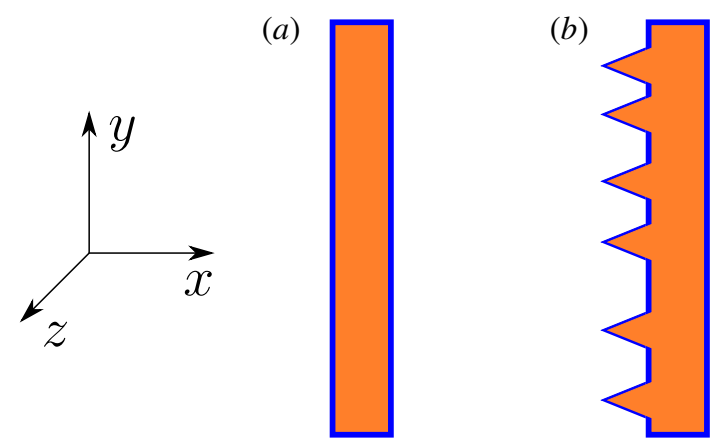

(c)

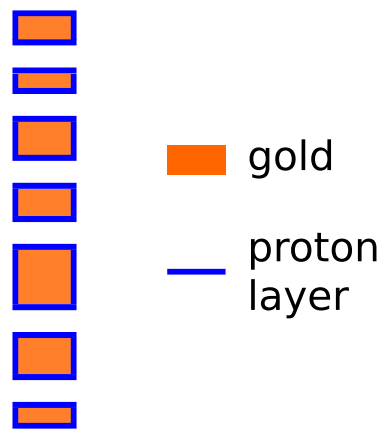

FIGURE 1. Schematic representation of the flat foil $(a)$, nanocone $(b)$ and nanohole $(c)$ targets. The targets are irradiated by the laser pulse from the left under an incidence angle of $45^{\circ}$.

the SMILEI code (Derouillat et al. 2018). The considered structures are visualized in figure $1(a-c)$. The reference target is a flat foil of thickness $d=100 \mathrm{~nm}$. It is composed of cold gold atoms, assumed to be 11-times ionized, with an ion number density of $5.85 \times 10^{22} \mathrm{~cm}^{-3}$. Simulations with different ionization states of gold atoms (up to 29-times ionized) were also performed but did not result in significant differences in proton spectra. A $10 \mathrm{~nm}$ thin proton-electron plasma layer with a number density of $1.74 \times 10^{23} \mathrm{~cm}^{-3}$ is added on the foil surfaces to model the hydrogen contaminants.

The nanocone target, sketched in figure $1(b)$, is composed of the above-mentioned flat foil coated with a distribution of cones, each having an opening angle of $44^{\circ}$ and a variable base size of $b$ (specific values will be set below). The nanohole target, displayed in figure $1(c)$, consists of the reference foil pierced by holes of the same width and location as the above nanocones. The surfaces of both types of structured targets are coated with a proton layer.

For the initialization of the nanocones' positions, we choose the following model. Given the position $y_{1}$ of the first nanocone, the average distance $D=2.24 b-$ motivated by practical considerations for target manufacturing - and the distance spread parameter $s=D / 2$, the position of the $i$ th nanocone is set iteratively to $y_{i}=y_{i-1}+D+(u-1 / 2) s$ as long as $y_{i}<y_{\max }$, where $y_{\max }$ is the maximal position and $u$ is a uniformly distributed random number taking values from 0 to 1 . This leads to an average areal cone density of $\rho=b / D=44 \%$. The targets are located at $x=20 \mu \mathrm{m}$.

The $p$-polarized laser pulse has a wavelength of $\lambda=0.8 \mu \mathrm{m}$ and a maximum intensity of $I_{0}=5 \times 10^{19} \mathrm{~W} \mathrm{~cm}^{-2}$, corresponding to a normalized vector potential $a_{0}=4.8$ and a peak electric field of $194 \mathrm{GV} \mathrm{cm}^{-1}$. It has Gaussian space and time profiles with a focal spot of $5 \mu \mathrm{m}$ FWHM (full width half maximum) and a duration of $38 \mathrm{fs}$ FWHM. It is incident on the target from the lower left-hand side, at $45^{\circ}$ from the surface normal. The peak intensity of the laser pulse reaches the foil after $175 \mathrm{fs}$ in the simulation. In the following, we will set this as the time reference $t=0$.

The numerical discretization of the simulations is $\delta x=\delta y=5 \mathrm{~nm}$ and $\delta t=11.7$ as. We use 100 macro-particles per cell and per species in the bulk plasma, while the surface proton-electron layers are represented by 1000 macro-particles per cell and per species. 

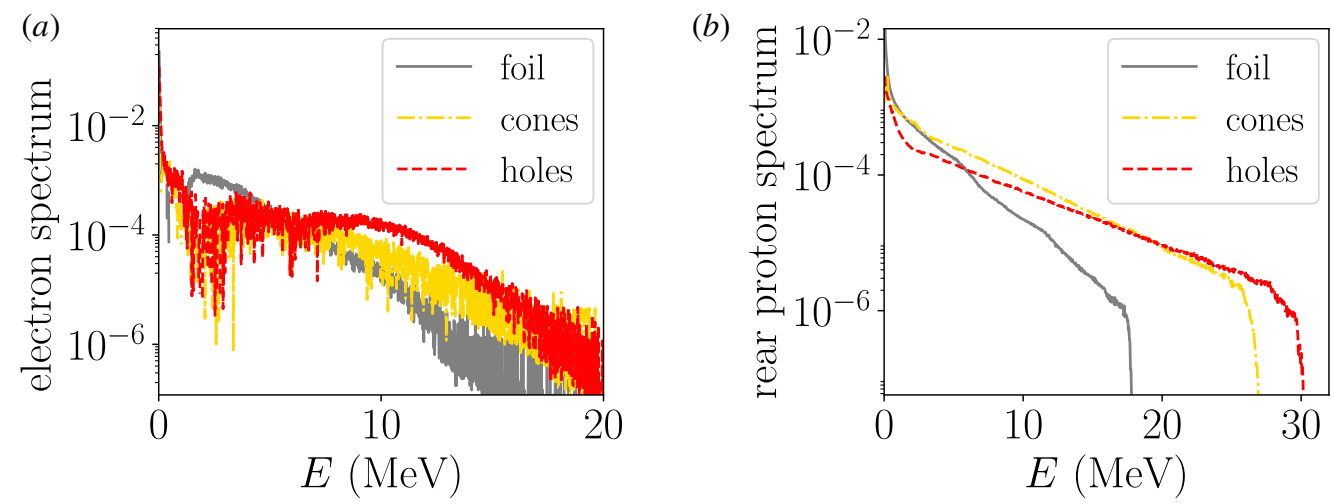

FIGURE 2. Energy spectra of electrons $(a)$ and rear-side protons $(b)$ from a flat foil (solid black), a nanocone (dashed-dotted yellow) and a nanohole (dashed red) target of $d=100 \mathrm{~nm}$ thickness. In $(a)$, only electrons located in the vacuum behind the target backside are considered. Electron spectra are recorded at $t=175 \mathrm{fs}$ and proton spectra at $t=455$ fs.

\section{Enhancement of electron heating and ion acceleration}

Periodic cone structures have been shown to enhance proton acceleration due to a modification of electron trajectories, hence maximizing laser absorption (Blanco et al. 2017). It is thus interesting to investigate whether completely relaxing the restriction of periodicity would impact the acceleration process. Here, we consider a non-periodic arrangement of the structures. The cone and hole-base size in this section is fixed to be $b=300 \mathrm{~nm}$.

The strength of the rear-side sheath field that underpins TNSA, and which therefore determines the efficiency of the latter, is controlled by the energy density of the lasergenerated hot electrons (Daido et al. 2012; Macchi et al. 2013). Figure 2(a) plots the energy spectrum of the electrons located in the vacuum region behind the rear side of the target, recorded at $t=175$ fs. Those electrons mainly account for the generation of the sheath electric field in the early stages of TNSA, when the approximation of the one-dimensional plasma expansion holds. Compared with the flat foil, both structured targets lead to a significantly increased number of relativistic electrons above $7 \mathrm{MeV}$, with the nanohole target yielding the largest enhancement - by approximately an order of magnitude.

A similar behaviour is found for the rear-side proton energy spectra, as plotted in figure $2(b)$ at $t=455 \mathrm{fs}$. Both nanocone and nanohole targets give rise to much enhanced TNSA: the best performance is observed using nanoholes, with an almost doubled proton cutoff energy compared to that from the flat foil ( $\sim 30 \mathrm{MeV}$ protons versus $\sim 17 \mathrm{MeV}$ ).

Figure 3 demonstrates that the electron and proton spectra are almost the same for periodic and non-periodic targets, consistent with the conclusion reached by Klimo et al. (2011) when investigating irregular spherical structures. It is thus to be expected that the individual structuring units, rather than their periodic arrangement (possibly leading to the excitation of surface plasma waves), are responsible for the enhancement. This is a favourable result from an experimental perspective since it reduces the target manufacturing constraints.

Moreover, figure $3(a, b)$ presents the electron and rear-proton energy spectra obtained for a flat foil (blue solid line) of reduced thickness $(d=55 \mathrm{~nm})$ such that it contains 
(a)

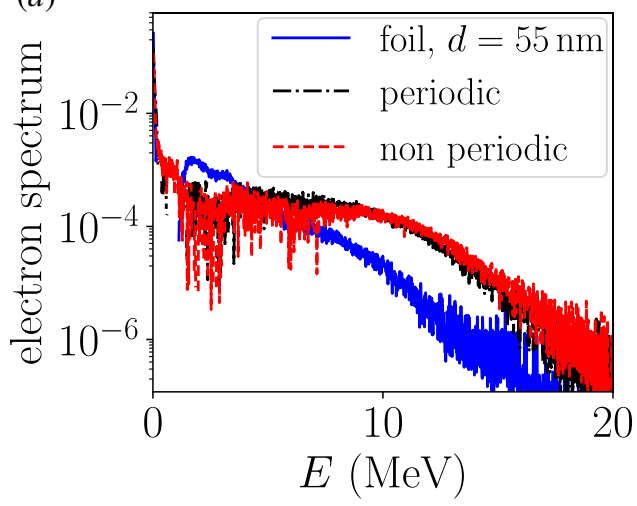

(b)

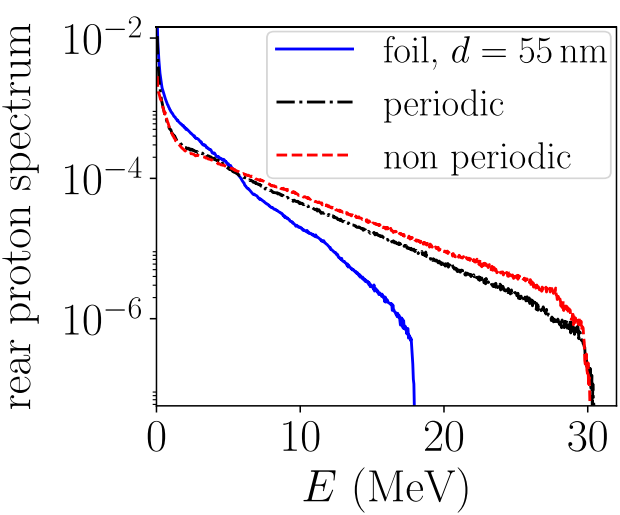

FIGURE 3. Energy spectra of the electrons $(a)$ and rear-protons $(b)$ from a flat foil (solid blue), a $d=100 \mathrm{~nm}$ periodic nanohole target (dashed-dotted black) and a $d=100 \mathrm{~nm}$ non-periodic nanohole target (dashed red). In $(a)$, only electrons located in the vacuum behind the target backside are considered. Electron spectra are recorded at $t=175 \mathrm{fs}$ and proton spectra at $t=455$ fs.

the same total amount of matter as the $d=100 \mathrm{~nm}$ nanohole targets, whether periodic or not. This thinner flat foil produces particle energy spectra very similar to the $d=100 \mathrm{~nm}$ foil; hence, the enhanced performance of the nanohole targets cannot be ascribed to the direct effect of their reduced volume (or area in two dimensions) on the electron kinetic energy density (which would naturally increase assuming the same amount of laser energy is converted into hot electrons), but rather results from a strongly modified hot-electron dynamics.

In order to gain insight into the electron energization process in the nanostructured target, we record the maximum Lorentz factor $\gamma$ reached by each electron during the simulation, and plot its locally averaged value as a function of the initial electron position (figure $4 a-c$ ). In the case of the flat foil (figure $4 a$ ), the resulting map shows that, as expected, most of the accelerated electrons originate from a $\sim 25 \mathrm{~nm}$-thick surface layer at the directly irradiated front side of the target (with mean energies $\sim 6 \mathrm{MeV}$ being reached), and that rear-side electrons undergo negligible acceleration. In the case of the nanohole (figure $4 b$ ) and nanocone (figure $4 c$ ) targets, some of the highest energy electrons stem from additional regions, namely the nanohole walls and the nanocone sides. The nanostructuring of the target surface therefore increases the effective interaction area, leading to a larger number of hot electrons. The mean energy $(\sim 10 \mathrm{MeV})$ reached by these electrons is also significantly larger than in the flat foil. These effects are supported by the local enhancement of the electrostatic field which appears when using nanostructured targets. The electrostatic field is indeed strongly enhanced at the corners of the nanoholes and at the tips of the nanocones, which correspond to the surfaces from which the most energetic electrons arise. Note that the value of the electrostatic field in these regions becomes of the same order as the laser field (figure $4 d-f$ ), which is also favourable for electron acceleration (Paradkar, Krasheninnikov \& Beg 2012). Interestingly, when the target is nanostructured, the change in the laser field pattern on the front side of the target indicates a decrease in the laser reflection compared with the flat foil. This is confirmed by the reflection coefficient, which drops from $C_{R_{f}}=0.67$ to $C_{R_{c}}=0.15$ when adding the nanocones. While the flat and nanocone targets remain 

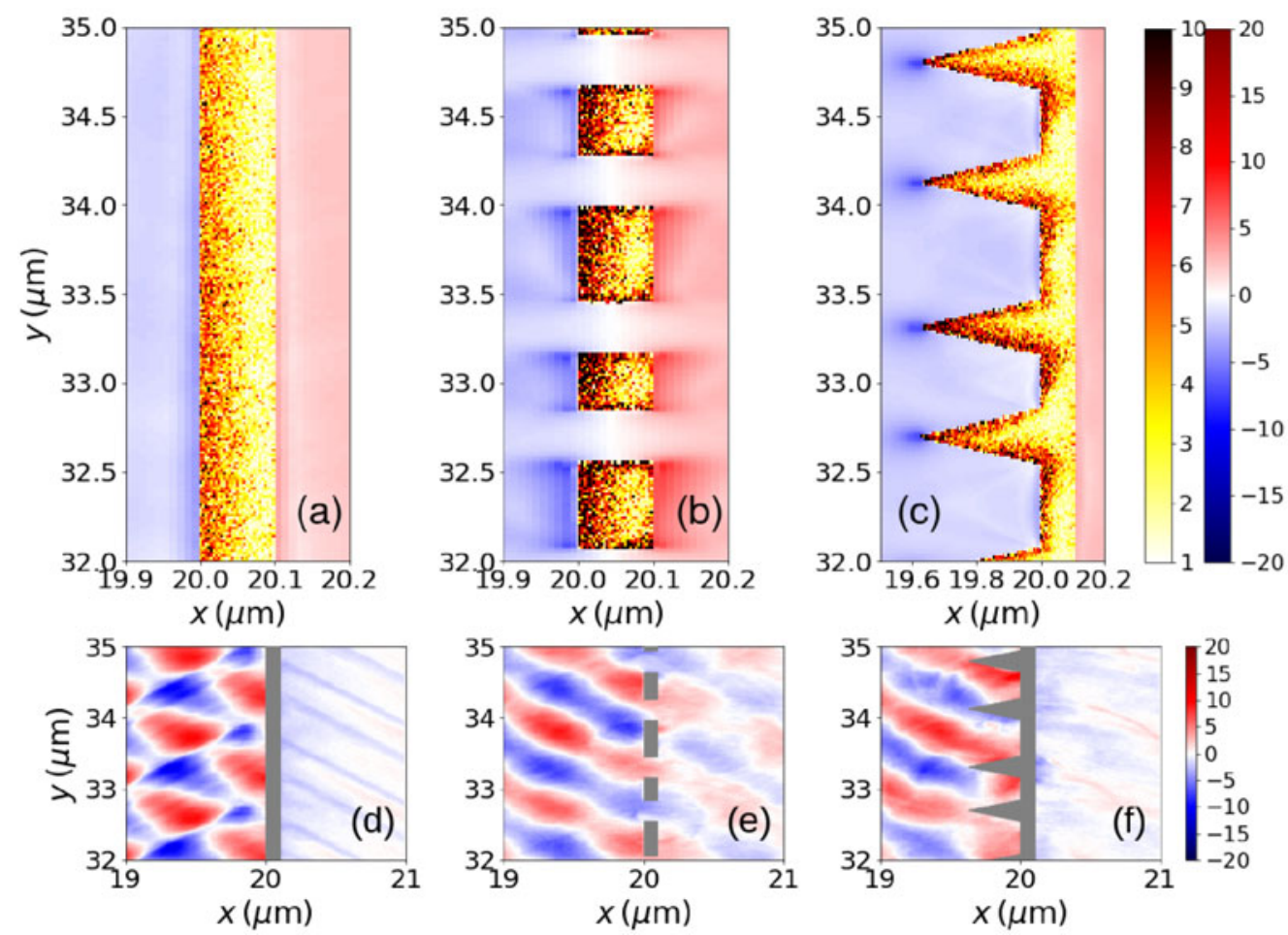

FIgURE 4. (a)-(c) Maximum $\gamma$ factor (locally averaged over the initial particle distribution) reached by electrons as a function of their initial position (yellow-red) and longitudinal electric field $E_{x}$ (units $m_{e} c \omega_{0} / e$, with $c$ the speed of light, $\omega_{0}$ the laser frequency, $m_{e}$ the electron mass and $e$ the elementary charge) at $t=0$, averaged over a laser period $T_{0}$ (blue-red). $(d)-(f)$ Magnetic field $B_{z}$ (units $m_{e} \omega_{0} / e$ ) at $t=0$. The initial target densities are indicated in light grey, and correspond to $100 \mathrm{~nm}$-thick flat $(a, d)$ nanohole $(b, e)$ and nanocone targets $(c, f)$ and the center of the laser spot on target is localized at $y=33.5 \mu \mathrm{m}$.

essentially opaque (with a transmission coefficient $C_{T_{f, c}}<0.02$ ), the laser light is partially transmitted through the nanoholes $\left(C_{T_{h}}=0.13\right)$, as is evident from figure 4 . Consequently, the absorption coefficient is lower in nanohole $\left(C_{A_{h}}=0.66\right)$ than in nanocone targets $\left(C_{A_{c}}=0.83\right)$. As further discussed below, this finding somewhat contradicts the widely shared notion that the absorbed laser energy fraction is the single figure of merit for TNSA.

This general behaviour can be complemented by examining individual electron trajectories. We focus on those high-energy electrons breaking through the target rear side, and therefore contributing to the accelerating sheath field. For this reason, figure 5 plots the trajectories of a sample of the most energetic electrons in both the flat and nanohole targets - with a lower energy cutoff for the selection of $12 \mathrm{MeV}$ in the nanohole target (figure $5 a$ ) and of $5 \mathrm{MeV}$ in the flat target case (figure $5 b$ ). Only those electrons that are located at a longitudinal position $x \geqslant 48 \mu \mathrm{m}$ at $t=175 \mathrm{fs}$ are selected. The colour of each trajectory is indexed on the rate of change of electron energy in the local electromagnetic fields. It can be seen that, on average, higher values are attained in the nanohole target. While the selected electrons mainly exhibit acceleration in the front- and rear-side vacuum regions, sizable energy transfer is also 
(a)

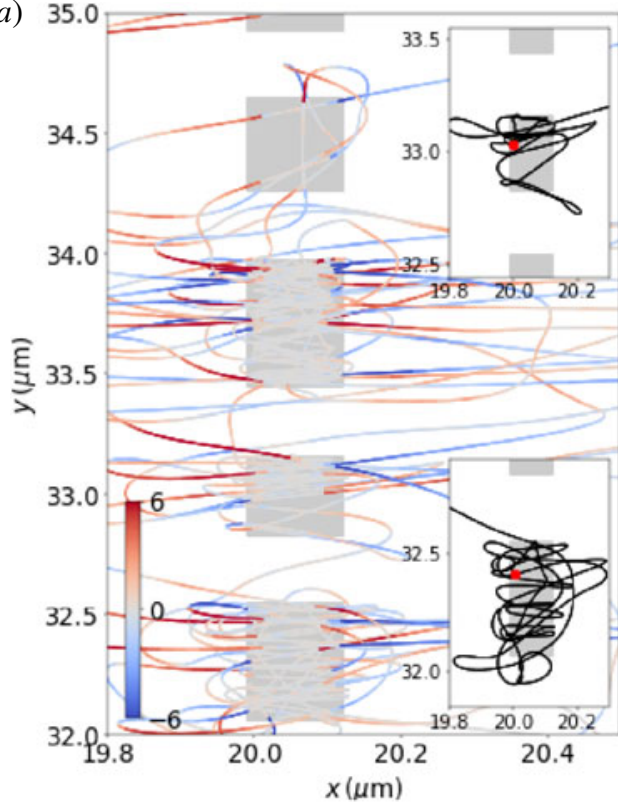

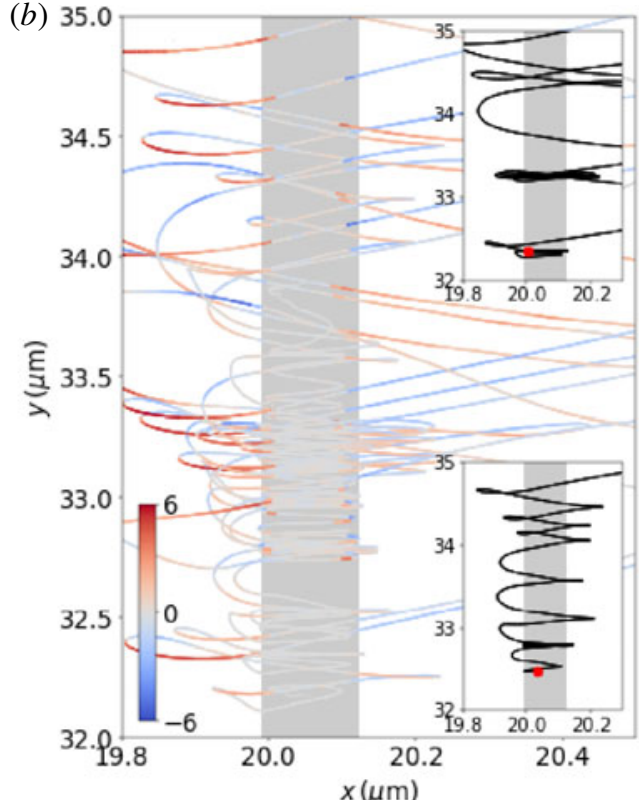

FIGURE 5. Trajectories of a few energetic electrons in the nanohole target $(a)$ and the flat target $(b)$. These electrons are selected randomly amongst those verifying $x>48 \mu \mathrm{m}$ at $t=175 \mathrm{fs}$, and as long as they have reached a threshold energy $E_{\mathrm{th}}$ during the simulation, with $E_{\mathrm{th}}=12 \mathrm{MeV}$ in the nanohole case and $E_{\mathrm{th}}=5 \mathrm{MeV}$ in the flat target case. The initial target density is indicated in light grey. The colour of the trajectories (blue-red) represents the rate of change $\left(\left(p_{x} E_{x}+p_{y} E_{y}\right) / \gamma\right)$ of electron energy (units $\left.m_{e}^{2} c^{2} \omega_{0} / e\right)$. Two trajectories of single electrons (black) are represented for each case in the different insets. The red dots indicate the initial positions of these particles.

seen within the cavities, which is consistent with the laser being partially transmitted through the nanoholes (see figure $4 e$ ).

The sub-micron dense regions that make up the nanohole targets effectively behave as mass-limited targets (Psikal et al. 2008; Buffechoux et al. 2010), leading to efficient electrostatic confinement of the hot electrons. This is evidenced by the single particle trajectories displayed in the insets of figure 5: the nanohole target causes the electron to recirculate in both longitudinal (across the front and rear sides of the target) and transverse (across the nanohole walls) directions. A favourable consequence of this is a longer effective laser-electron interaction time. Also, the laser-electron interaction occurs under various geometrical conditions, and so with increased degrees of freedom. This results in a more complete exploration of the phase space, which ultimately allows the electrons to be accelerated to higher energies. Finally, being prevented from leaving the laser-irradiated region, the hot electrons are able to sustain a strong sheath field over longer times. The confinement of the hot electrons entails a reduced transverse extent of the sheath field, and therefore of the expanding proton cloud. This can be seen in figure 6, which shows the boundary of the proton cloud as resulting from the three target types, the nanohole target giving rise to a more narrow proton distribution. Compared to nanocone targets, the more localized and intense sheath fields induced in nanohole targets translate into lower numbers of accelerated protons but of higher energies, despite lower laser absorption. A drawback 


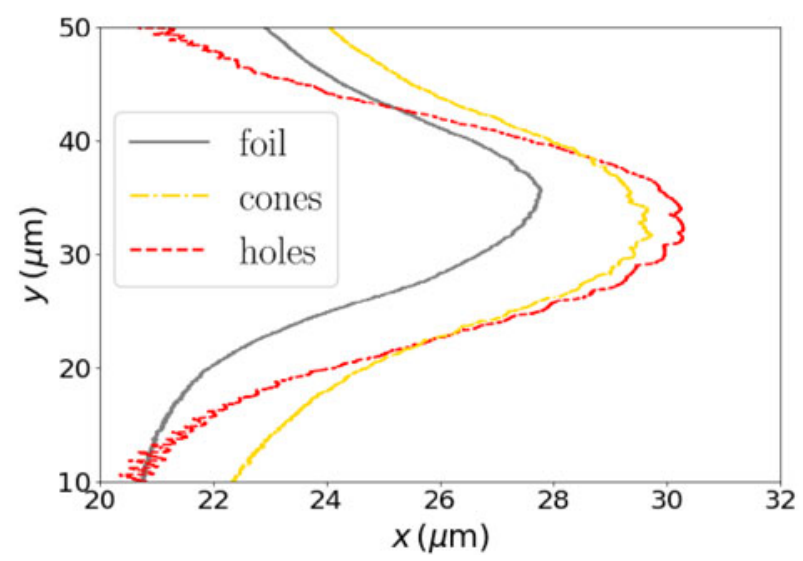

FIgURE 6. Position of the front of the accelerating rear-proton layer at $t=175 \mathrm{fs}$ for a flat foil (solid black), a nanocone (dashed-dotted yellow) and a nanohole (dashed red) targets of $d=100 \mathrm{~nm}$ thickness.

of nanohole targets, however, is the significant transverse sheath fields which build up at the sharp transverse vacuum-plasma interfaces, and tend to increase the divergence of the proton beam (measured to be approximately twice higher in nanohole targets than in flat foils).

\section{Parametric scan for nanohole targets}

We now perform a parametric scan where we vary the foil thickness $d$ and hole diameter $b$. Henceforth, we consider only the cases where the hole diameter is at least as large as the foil thickness, i.e. $b \geqslant d$.

Figure $7(a)$ presents the spectra of the rear-side electrons for foil thicknesses from 100 to $600 \mathrm{~nm}$ and various hole diameters. One can see that the electrons from the flat foil (grey line) reach lower energies than electrons from perforated foils, whatever the hole diameter. Especially above $5 \mathrm{MeV}$, perforated targets produce more high-energy electrons. The enhancement is very similar for hole sizes from 100 to $600 \mathrm{~nm}$.

While we are mostly interested in the protons accelerated from the target back side, we also plot for completeness the energy spectra of the protons originating from the front side. Figure $7(b, c)$ presents the corresponding front $(b)$ and rear $(c)$ proton spectra. In both cases, the structuring significantly enhances the proton cutoff energies. While a $100 \mathrm{~nm}$ hole size already enhances the proton cutoff energy by approximately $40 \%-50 \%$, the largest enhancements are reached for 300 to $600 \mathrm{~nm}$ diameter holes.

When increasing the gold foil thickness, the trends remain the same (see figure $7 d-i$ ). The presence of the holes increases the number of high-energy electrons and boosts the proton energy by approximately a factor of 2 . The thinner the gold foil, the higher the proton energies. However, while this nanohole structuring can be used for all target thicknesses, the relative improvement is slightly more pronounced for thicker foils (by $95 \%$ at $d=300 \mathrm{~nm}$ versus $70 \%$ at $d=100 \mathrm{~nm}$ ).

The PIC simulation results suggest that as long as the parameters are in the abovedescribed range leading to enhancement, they can be freely chosen depending on other experimental constraints, e.g. ease of handling and fabrication.

Note that our simulations consider sharp-gradient targets, meaning that they do not address the possible influence of finite preplasmas caused by laser prepulses. 
(a)

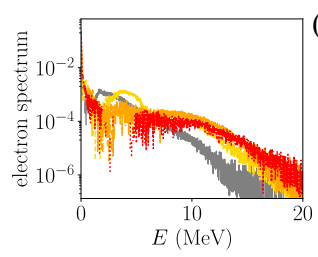

(d)

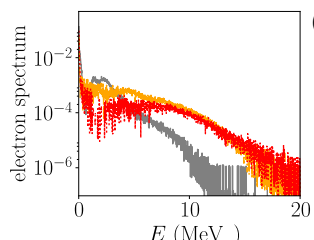

(g)

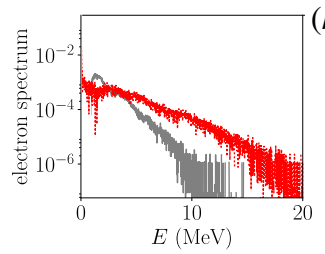

(b)

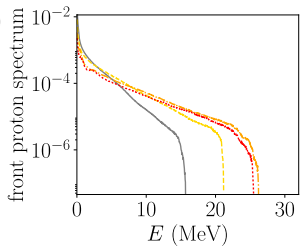

(e)
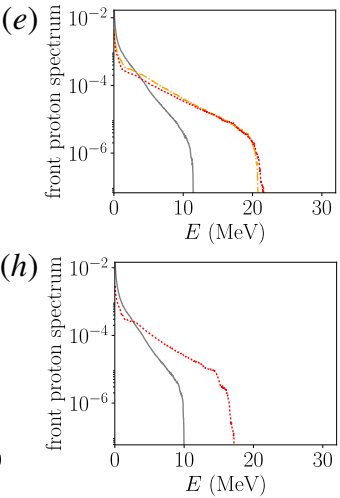

(c)

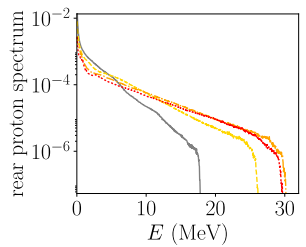

$(f)$

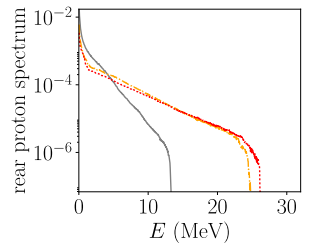

(i)

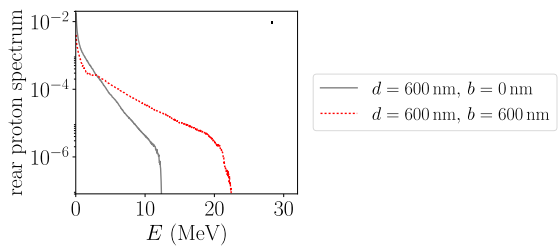

FIGURE 7. Particle density spectra of the rear-side electrons $(a, d, g)$, front-side protons $(b, e, h)$ and rear-side protons $(c, f, i)$ from nanohole targets. The electron spectra are presented at $t=175 \mathrm{fs}$, the front proton spectra at $t=315 \mathrm{fs}$ and the rear proton spectra at $t=455 \mathrm{fs}$. The foil thickness is $d=100 \mathrm{~nm}$ in $(a-c), d=300 \mathrm{~nm}$ in $(d-f)$, $d=600 \mathrm{~nm}$ in $(g-i)$. The nanohole diameter is varied in the range $0 \leqslant b \leqslant 600 \mathrm{~nm}$, as indicated in the legend of each row.

(a)

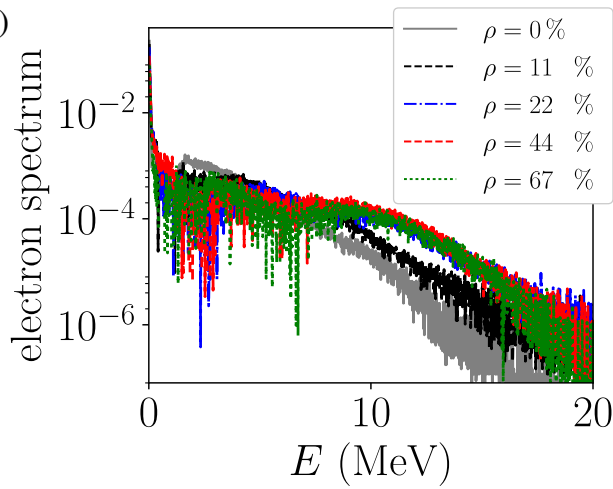

(b)

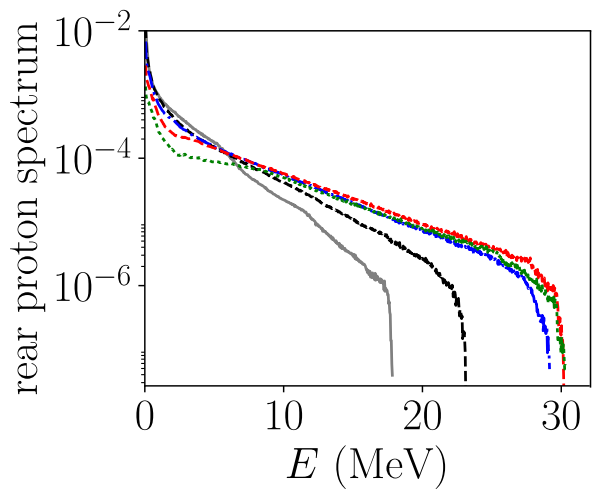

FIGURE 8. Rear-side electron and proton energy spectra under conditions similar to those of figure 2 , but with different hole densities $\rho$ according to the legends in $(a)$ and $(b)$.

The generation of a significant preplasma would modify the picture: the holes might be filled with electrons before the arrival of the main pulse, thus suppressing the benefit of the target structuring. Larger hole diameters may then be preferable under actual experimental conditions.

The areal density of the holes can be expected to play a role in enhancing TNSA. Figure 8 displays the energy spectra of the rear-side electrons and protons obtained from $100 \mathrm{~nm}$ thick nanohole targets of nanohole density varying in the range $0 \leqslant \rho \leqslant 67 \%$. An optimum electron heating and proton acceleration is reached when 
almost half of the surface area is covered by the holes. However, already for a density of approximately $20 \%$, a notable increase in the cutoff energy is reached.

The optimum is likely formed by two counteracting effects. On the one hand, when increasing the hole density, the mechanisms described in $\$ 3$ further develop: more electrons can get an energy boost due to the increase in the interaction surface and recirculation. On the other hand, raising the hole density reduces the effective target volume, eventually resulting in higher laser transmission, and therefore weaker lasertarget coupling. To mitigate the latter effect, one could use thicker foils instead of the considered $100 \mathrm{~nm}$ thin foils. However, this solution is limited by the fact that the proton cutoff energy tends to be reduced when the nanohole target gets thicker - in the same manner as for the standard flat-foil target.

\section{Conclusion}

In summary, by means of two-dimensional PIC simulations, we investigate laser-driven proton acceleration from non-periodic nanohole and nanocone targets. We demonstrate a significant increase in the proton cutoff energy in both types of structured targets compared to flat foils. This better performance is found to originate from several factors: an enlarged effective interaction surface between the laser and the target, a larger number of degrees of freedom in the electron dynamics and a local intensification of the charge-separation fields. In the case of nanohole targets, we identify a large parameter space in terms of hole diameter, foil thickness and hole areal density yielding significant enhancement of the accelerated proton spectra. The specific nanohole geometry yields additional control through the limitation of the hot electrons' transverse expansion. Interestingly, the enhancement of the ion acceleration does not stem only from the increased laser-to-electron conversion efficiency, since nanohole targets yield higher proton energies than nanocone targets despite a lower laser absorption.

Finally, our results show that the production of structured targets for improved ion acceleration can be relaxed to non-periodic structures with a relatively low areal density of structuring units.

\section{Acknowledgements}

The authors acknowledge fruitful discussions with $\mathrm{L}$. Yi and the rest of the PLIONA team. This work was supported by the Knut and Alice Wallenberg Foundation, the European Research Council (ERC-2014-CoG grant 647121) and by the Swedish Research Council, grants no. 2016-05012 and no. 2017-04684. The simulations were performed on resources provided by GENCI (project A0060507594) and at Chalmers Centre for Computational Science and Engineering (C3SE) provided by the Swedish National Infrastructure for Computing (grants SNIC2018-2-13 and SNIC2018-3-297).

\section{REFERENCES}

Andreev, A., Kumar, N., Platonov, K. \& Pukhov, A. 2011 Efficient generation of fast ions from surface modulated nanostructure targets irradiated by high intensity short-pulse lasers. Phys. Plasmas 18 (10), 103103.

Andreev, A., Platonov, K., Braenzel, J., Lübcke, A., Das, S., Messaoudi, H., Grunwald, R., Gray, C., McGlynn, E. \& SChNÜRER, M. 2016 Relativistic laser nano-plasmonics for effective fast particle production. Plasma Phys. Control. Fusion 58 (1), 014038. 
Blanco, M., Flores-Arias, M. T., Ruiz, C. \& Vranic, M. 2017 Table-top laser-based proton acceleration in nanostructured targets. New J. Phys. 19, 033004.

Blanco, M., Flores-Arias, M. \& VRAnic, M. 2019 Suitability and robustness of triangular nanostructured targets for proton acceleration. arXiv:1902.05641.

Brantov, A. \& BYchenkov, V. Y. 2013 Laser-triggered proton acceleration from micro-structured thin targets. Contributions Plasma Phys. 53 (10), 731-735; arXiv:https://onlinelibrary.wiley. com/doi/pdf/10.1002/ctpp.201310046.

Brenner, C. M., Robinson, A. P. L., Markey, K., Scott, R.H.H., Gray, R. J., Rosinski, M., Deppert, O., BADZiaK, J., BAtani, D., DAVIES, J.R. et al. 2014 High energy conversion efficiency in laser-proton acceleration by controlling laser-energy deposition onto thin foil targets. Appl. Phys. Lett. 104 (8), 081123.

Buffechoux, S., Psikal, J., Nakatsutsumi, M., Romagnani, L., Andreev, A., Zeil, K., Amin, M., Antici, P., Burris-Mog, T., Compant-La-Fontaine, A. et al. 2010 Hot electrons transverse refluxing in ultraintense laser-solid interactions. Phys. Rev. Lett. 105, 015005 .

Bulanov, S. V. \& Khoroshkov, V. S. 2002 Feasibility of using laser ion accelerators in proton therapy. Plasma Phys. Rep. 28, 453-456.

Ceccotti, T., Floquet, V., Sgattoni, A., Bigongiari, A., Klimo, O., Raynaud, M., Riconda, C., Heron, A., Baffigi, F., Labate, L. et al. 2013 Evidence of resonant surface-wave excitation in the relativistic regime through measurements of proton acceleration from grating targets. Phys. Rev. Lett. 111, 185001.

Daido, H., Nishiuchi, M. \& PirozhKov, A. S. 2012 Review of laser-driven ion sources and their applications. Rep. Prog. Phys. 75 (5), 056401.

Derouillat, J., Beck, A., Pérez, F., Vinci, T., Chiaramello, M., Grassi, A., Flé, M., Bouchard, G., Plotnikov, I., Aunai, N. et al. 2018 Smilei: a collaborative, open-source, multi-purpose particle-in-cell code for plasma simulation. Comput. Phys. Commun. 222, 351-373.

Fedeli, L., Formenti, A., Cialfi, L., Pazzaglia, A. \& Passoni, M. 2018 Ultra-intense laser interaction with nanostructured near-critical plasmas. Sci. Rep. 8 (1), 3834.

FERri, J., Siminos, E. \& FÜLÖP, T. 2019 Enhanced target normal sheath acceleration using colliding laser pulses. Commun. Phys. 2, 40.

Habs, D., Thirolf, P. G., Gross, M., Allinger, K., Bin, J., Henig, A., Kiefer, D., Ma, W. \& SCHREIBER, J. 2011 Introducing the fission-fusion reaction process: using a laser-accelerated Th beam to produce neutron-rich nuclei towards the $n=126$ waiting point of the $r$-process. Appl. Phys. B 103 (2), 471-484.

Higginson, A., Gray, R. J., King, M., Dance, R. J., Williamson, S. D. R., Butler, N. M. H., Wilson, R., Capdessus, R., Armstrong, C., Green, J. S. et al. 2018 Near-100 MeV protons via a laser-driven transparency-enhanced hybrid acceleration scheme. Nature Commun. 9 (1), 724.

Kaluza, M., Schreiber, J., Santala, M. I. K., Tsakiris, G. D., Eidmann, K., Meyer-ter VeHn, J. \& WitTE, K. J. 2004 Influence of the laser prepulse on proton acceleration in thin-foil experiments. Phys. Rev. Lett. 93, 045003.

Klimo, O., Psikal, J., Limpouch, J., Proska, J., Novotny, F., Ceccotti, T., Floquet, V. \& KAWATA, S. 2011 Short pulse laser interaction with micro-structured targets: simulations of laser absorption and ion acceleration. New J. Phys. 13 (5), 053028.

Langhammer, C., Kasemo, B. \& Zoric, I. 2007 Absorption and scattering of light by Pt, Pd, $\mathrm{Ag}$, and Au nanodisks: absolute cross sections and branching ratios. J. Chem. Phys. 126 (19), 194702.

Macchi, A., Borghesi, M. \& PAssoni, M. 2013 Ion acceleration by superintense laser-plasma interaction. Rev. Mod. Phys. 85, 751.

Margarone, D., Klimo, O., Kim, I. J., ProkŮPeK, J., Limpouch, J., Jeong, T. M., Mocek, T., PŠIKAL, J., KIM, H. T., ProšKA, J. et al. 2012 Laser-driven proton acceleration enhancement by nanostructured foils. Phys. Rev. Lett. 109, 234801. 
Neely, D., Foster, P., Robinson, A., Lindau, F., Lundh, O., Persson, A., Wahlström, C.-G. \& McKenna, P. 2006 Enhanced proton beams from ultrathin targets driven by high contrast laser pulses. Appl. Phys. Lett. 89 (2), 021502.

Nodera, Y., Kawata, S., Onuma, N., Limpouch, J., Klimo, O. \& KikUChi, T. 2008 Improvement of energy-conversion efficiency from laser to proton beam in a laser-foil interaction. Phys. Rev. E 78, 046401.

Nuter, R., Gremillet, L., Combis, P., Drouin, M., Lefebvre, E., Flacco, A. \& Malka, V. 2008 Influence of a preplasma on electron heating and proton acceleration in ultraintense laser-foil interaction. J. Appl. Phys. 104, 103307.

Ogura, K., Nishiuchi, M., Pirozhkov, A. S., Tanimoto, T., Sagisaka, A., Esirkepov, T. Z., Kando, M., Shizuma, T., Hayakawa, T., Kiriyama, H. et al. 2012 Proton acceleration to $40 \mathrm{MeV}$ using a high intensity, high contrast optical parametric chirped-pulse amplification/ti: sapphire hybrid laser system. Opt. Lett. 37 (14), 2868-2870.

Paradkar, B. S., Krasheninnikov, S. I. \& Beg, F. N. 2012 Mechanism of heating of pre-formed plasma electrons in relativistic laser-matter interaction. Phys. Plasmas 19 (6), 060703.

Psikal, J., Grym, J., Stolcova, L. \& Proska, J. 2016 Hollow target for efficient generation of fast ions by ultrashort laser pulses. Phys. Plasmas 23 (12), 123121.

Psikal, J., Tikhonchuk, V. T., Limpouch, J., Andreev, A. A. \& Brantov, A. V. 2008 Ion acceleration by femtosecond laser pulses in small multispecies targets. Phys. Plasmas 15 (5), 053102.

Romagnani, L., Fuchs, J., Borghesi, M., Antici, P., Audebert, P., Ceccherini, F., Cowan, T., Grismayer, T., KAR, S., MACCHI, A. et al. 2005 Dynamics of electric fields driving the laser acceleration of multi-MeV protons. Phys. Rev. Lett. 95, 195001.

Sgattoni, A., Sinigardi, S., Fedeli, L., Pegoraro, F. \& Macchi, A. 2015 Laser-driven Rayleigh-Taylor instability: plasmonic effects and three-dimensional structures. Phys. Rev. E 91, 013106.

Snavely, R. A., Key, M. H., Hatchett, S. P., Cowan, T. E., Roth, M., Phillips, T. W., Stoyer, M. A., Henry, E. A., Sangster, T. C., Singh, M. S. et al. 2000 Intense highenergy proton beams from petawatt-laser irradiation of solids. Phys. Rev. Lett. 85, 2945-2948.

Wagner, F., Deppert, O., Brabetz, C., Fiala, P., Kleinschmidt, A., Poth, P., Schanz, V. A., Tebartz, A., Zielbauer, B., Roth, M. et al. 2016 Maximum proton energy above $85 \mathrm{MeV}$ from the relativistic interaction of laser pulses with micrometer thick $\mathrm{CH}_{2}$ targets. Phys. Rev. Lett. 116, 205002.

Wilks, S. C., Langdon, A. B., Cowan, T. E., Roth, M., Singh, M., Hatchett, S., Key, M. H., Pennington, D., MacKinnon, A. \& Snavely, R. A. 2001 Energetic proton generation in ultra-intense laser-solid interactions. Phys. Plasmas 8 (2), 542-549.

Yu, J., Zhou, W., Jin, X., CaO, L., ZhaO, Z., Hong, W., LI, B. \& GU, Y. 2012 Improvement of proton energy in high-intensity laser-nanobrush target interactions. Laser Part. Beams 30 (2), 307-311.

Zigler, A., Eisenman, S., Botton, M., Nahum, E., Schleifer, E., Baspaly, A., Pomerantz, I., Abicht, F., Branzel, J., Priebe, G. et al. 2013 Enhanced proton acceleration by an ultrashort laser interaction with structured dynamic plasma targets. Phys. Rev. Lett. 110, 215004.

Zigler, A., Palchan, T., Bruner, N., Schleifer, E., Eisenmann, S., Botton, M., Henis, Z., Pikuz, S. A., FAenov, A. Y., Gordon, D. et al. 2011 5.5-7.5 MeV proton generation by a moderate-intensity ultrashort-pulse laser interaction with $\mathrm{H}_{2} \mathrm{O}$ nanowire targets. Phys. Rev. Lett. 106, 134801. 